\title{
LA RINASCITA AL TERMINE DEL VIAGGIO. SEGUENDO I 'MOVIMENTI' DI CAMERE SEPARATE DI PIER VITTORIO TONDELLI
}

\section{CRISTIANA FURLAN}

Riassunto: "La rinascita al termine del viaggio. Seguendo i movimenti di Camere separate" esamina l'interazione fra il tema del viaggio e quello della rigenerazione nel romanzo di Pier Vittorio Tondelli. L'articolo procede investigando le modalità scelte dall'autore per descrivere il personale sviluppo cognitivo del protagonista e, contemporaneamente, proponendo una lettura in chiave etica delle conseguenze di tale sviluppo. Tale approccio è supportato dalla considerazione che i momenti cruciali del romanzo sono quelli in cui il protagonista, per effetto della dinamica morte-rinascita, si percepisce come essere duplice, ovvero come padre e figlio, Sé ed Altro. L'analisi della dimensione etica si propone di approfondire quest'ultimo rapporto, esaminandolo nei termini proposti da Emmanuel Lévinas che vede nella relazione con l'alterità non un momento di reciproca soppressione o annullamento, bensì una possibilità conoscitiva della diversità senza che quest'ultima venga eliminata a vantaggio del Sé.

Fidarsi di ciò che un autore afferma a proposito dei propri romanzi non è mai una buona idea per un critico. La chiave interpretativa suggerita di solito non risulta completamente soddisfacente. Nel caso di Pier Vittorio Tondelli, però, c'è almeno un suggerimento che si può cogliere in sede di discussione critica. In un'intervista rilasciata a Fulvio Panzeri, a proposito di Camere separate lo scrittore dichiarava:

Più o meno la narrazione è conclusa in ogni movimento, un po' come la musica minimale o ambientale. C'è sempre la stessa nota, lo stesso gruppo di note, che si riproducono, quasi in circolo. Sembra che non cambi niente invece è un modo per scavare... Alla fine ti cambia la partitura. Questa è stata l'idea del libro. (Panzeri, Pier Vittorio Tondelli, 51)

Il paragone proposto da Tondelli fra i motivi contenuti nel suo romanzo ed il modo in cui le note vengono utilizzate in alcuni tipi di musica, funziona perfettamente1. La narrazione segue infatti lo stesso tipo di procedi-

\footnotetext{
${ }^{1}$ L'idea di una struttura narrativa che si rifa ad un movimento musicale non è completamente nuova in Tondelli. Già in Rimini, Bruno May scrive pagine che "assomiglino ad una partitura musicale" (Tondelli, Rimini, 645).
} 
mento: ruotando in modo apparentemente statico attorno alla storia d'amore fra Leo e Thomas, essa attraversa lentamente tutta la vita di Leo ripercorrendo le tappe importanti della sua esistenza prima e dopo l'incontro con Thomas, ma anche quelle successive alla morte di quest'ultimo. L'apparente indipendenza di ogni movimento quindi, si spiega in quanto ognuno di essi è un momento di un percorso, che in sè è concluso ma non chiuso, e va di conseguenza considerato, o ascoltato, come parte di un insieme più ampio.

Questa particolare struttura testuale, da un lato giustifica l'organizzazione in movimenti anziché in capitoli, dall'altro amplia le possibilità interpretative del sostantivo movimento. Movimento, infatti, in un'accezione più comune, ovvero meno specifica rispetto al campo semantico di riferimento, nel caso suggerito da Tondelli quello musicale, significa più semplicemente spostamento. Esso va inteso quindi in senso più vasto, perchè non si tratta solo di un'allusione ad un tipo di musica, ma è lo spostamento fisico che Leo deve fare per compiere il viaggio. Il movimento del viaggio, sempre per rimanere all'interno dell'interpretazione suggerita da Tondelli, è l'atto necessario a Leo per scavare. Uno dei movimenti del protagonista, infatti, è proprio quello di "scavare", metaforicamente, all'interno di se stesso. Quello che ne emerge a poco a poco sono alcuni dei nodi centrali della vita stessa: la nascita, la morte, l'amore, il dolore. Ne emerge anche un rapporto auto-rigenerativo di cui Leo è protagonista ed il cui punto di partenza è proprio il viaggio, interiore o fisico.

Ciò che questo articolo si propone è di prendere in esame l'interazione, a livello narrativo, fra la tematica del viaggio e quella della rigenerazione ${ }^{2}$. Più precisamente, quest'ultima va intesa proprio come rinascita, in quanto, come vedremo, i momenti più intensi della narrazione sono quelli in

${ }^{2}$ L'archetipo del viaggio, inteso come momento dell'esperienza umana dalle potenzialità rigenerative ha una lunghissima tradizione nella letteratura italiana e mondiale. In ambito italiano, l'esempio principe è indubbiamente la Divina commedia di Dante, benché nel suo caso la rinascita sia più legata ad una problematica di carattere spirituale che all'identità personale. In epoca contemporanea, un esempio di dinamica morte-rinascita che si sviluppa grazie al viaggio, e a cui fa seguito un'evoluzione dell'identità, si trova nel Fu Mattia Pascal di Luigi Pirandello. Nel romanzo pirandelliano, tuttavia, la sequenza rigenerativa che si conclude con una duplicità del soggetto, porta ad una stasi più che ad una effettiva rinascita. Lo sviluppo dell'identità resta immobilizzato nello sdoppiamento, ed il protagonista del romanzo sarà contemporaneamente Mattia Pascal e, paradossalmente, "fu Mattia Pascal," rimanendo imprigionato in un limbo psicologico e sociale dal quale non riesce a svincolarsi. 
cui Leo metaforicamente muore e rinasce; rinascita che assume le caratteristiche di un'auto-filiazione. Nel corso della vicenda Leo infatti si definisce più volte come contemporaneamente padre e figlio di se stesso. Una delle conseguenze di questa auto-filiazione è che Leo si percepisce come soggetto duplice, ovvero egli si identifica come sé e contemporaneamente come soggetto separato, cioè come "altro" con il quale deve costruire un rapporto ex-novo. Il tipo di rapporto che il protagonista metterà in atto con il nuovo sé, rappresenta lo sviluppo ed il naturale completamento del primo argomento discusso: una volta definite le modalità auto-rigenerative, occorre infatti indagare quali siano le conseguenze sul piano etico della relazione con l'altro.

Le sequenze morte-rinascita sono i momenti forti della crescita personale del protagonista, della sua maturazione; si vuole mettere l'accento sull'aspetto etico perciò, perché Leo è costretto, anche se spesso contro la sua volontà, a confrontarsi con le responsabilità nei confronti di quella nuova parte di sé che si sta formando. La dimensione fondamentale in cui l'etica si sviluppa è quella pratica, e vedremo che nel caso di Leo, se la rinascita è da intendersi con valore figurato, l'esito di quest'ultima ha però conseguenze sulla vita quotidiana del protagonista. Il trait-d'union fra il livello simbolico della nascita e quello della realtà, ovvero della prassi di vita, è costituito dal corpo stesso di Leo. I momenti in cui Leo sente di morire e di rinascere non sono vissuti solo a livello simbolico, come avvenimento metafisico, ma, al contrario, quando egli sentirà di sfiorare il nulla, la sensazione è principalmente fisica: è il corpo che sta morendo, o meglio, è il corpo che si sta separando da ciò che gli è intorno. Questa separazione, percepita prima come morte e poi come rinascita si conclude nel momento in cui il soggetto realizza la possibilità di differenziazione nell'unità.

La dimensione etica del romanzo verrà esaminata nei termini proposti dal filosofo franco-lituano Emmanuel Lévinas, il qualc, in Totalitè et infi$n i$, descrive in termini di non prevaricazione il rapporto fra il Sé e l'Altro. Egli propone un approccio non antagonistico in cui l'inclusione dell'altro nel mondo del Sé non si risolve con l'univoca soppressione della diversità che l'altro rappresenta, né, tantomeno, con l'eliminazione delle reciproche differenze. Egli sviluppa il suo discorso su base empirica esaminando occasioni e relazioni sociali che richiedono una "messa in gioco" del Sé. All'interno di questo quadro fenomenologico-relazionale, in cui Lévinas considera situazioni che vanno dall'eros alla dimora, viene inserita anche la relazione padre-figlio. Più precisamente Lévinas scrive: "La paternitè demeure une identification de soi, mais aussi une distinction dans l'unification [...]" (Totalité et infini, 229). Lévinas riferisce questa osservazione 
all'esperienza della paternità fisica, nella quale il genitore si riconosce idealmente nel proprio figlio, ma contemporaneamente vede in lui anche aspetti caratterizzanti la propria identità. Questo tipo di dinamica relazionale può essere applicato anche ad un ambito più strettamente soggettivo in quanto la distinzione a cui il filosofo fa riferimento, è riconoscibile anche nel momento in cui avviene una separazione interna all'individuo stesso. In quest'ultimo caso il soggetto percepisce il cambiamento che è avvenuto, o sta avvenendo in lui, per cui l'identità si trova ad essere quasi sdoppiata, ed il sé si riconosce come parte di un processo in evoluzione. In modo simile, il percorso di Leo, il movimento, si realizza attraverso un processo in cui la percezione di sé come essere umano in fieri si realizza nello sdoppiamento di una sola identità simboleggiato dal processo morte-rinascita. Il nuovo Leo sa di essere in parte lo stesso del giorno prima, ma, contemporaneamente, la differenziazione in atto gli fa guardare a sé come ad un individuo nuovo, semisconosciuto, l'altro. Gli sviluppi pratici, quindi suscettibili di una lettura etica, di questa evoluzione, si manifestano nella mediazione che Leo deve mettere in atto nella prassi quotidiana della sua vita, fra questo sé e l'altro, ovvero il nuovo Leo che si sa formando e verso il quale ha delle responsabilità.

La critica ha scarsamente preso in considerazione le questioni etiche che emergono da alcuni dei romanzi di Tondelli. In questo senso è vicino a Camere separate il precedente romanzo Rimini. In particolare, quella del giornalista Marco Bauer, è anche la storia di una maturazione personale che passa attraverso una presa di coscienza del proprio ruolo in dinamiche sociali dai risvolti in qualche caso illeciti. Bauer, arriva a Rimini con l'arroganza data dell'aver ottenuto piuttosto presto una promozione, ma si renderà conto, anche se troppo tardi, di essere stato usato e raggirato dai suoi stessi sottoposti. Nelle pagine finali il protagonista realizzerà la propria ingenuità, ma anche il fatto di non saper fare i conti con una realtà che ha meno scrupoli di quanti ne abbia lui. La sua scelta di cercare lavoro in un giornale di provincia corrisponde anche al non voler più essere un'importante voce giornalistica in una società in cui sembrano non esserci valori, ed in cui è possibile anche tacere un omicidio. Similmente a Camere separate anche alcune delle precedenti opere narrative di Tondelli si configurano come dei momenti di un processo di maturazione, se non come dei veri e propri riti di passaggio all'età adulta, come avviene, ad esempio, per i soldati in $P A O P A O$. In queste narrazioni, un elemento fondamentale è costituito dal senso di appartenenza: in Altri libertini, ad esempio, quasi tutti i protagonisti hanno un gruppo di riferimento. Questo bisogno di autoriconoscimento all'interno di una specifica comunità, che si percepisce 
come separata rispetto al resto della società, aveva importanti riflessi sul piano della relazione etica con la diversità. Nei primi romanzi il rapporto con "l'altro" aveva spesso una soluzione in senso antagonistico, la diversità diventava motivo di esclusione e separazione, ed il confronto si risolveva in un reciproco rifiuto ${ }^{3}$. In questi romanzi, il processo di crescita personale sembra non arrivare mai a completamento, ed i protagonisti restano immobilizzati in uno stato quasi adolescenziale; il bisogno ed insieme la sicurezza di avere un gruppo in cui riconoscersi non consentiva ai protagonisti l'individuazione di un ruolo personale all'interno della società. A differenza di Altri libertini o PAO PAO, in Camere separate, la maturazione arriva a completamento e si realizza su un altro livello, più personale senza essere più individualistico. Diversamente dagli altri personaggi tondelliani, la separazione che Leo avverte rispetto al mondo che lo circonda non può essere colmata dal sentirsi parte di uno specifico gruppo. Egli ha bisogno di trovare il suo posto "nel mondo", non in riferimento alla comunità omosessuale, di cui fa comunque parte, $\mathrm{ma}$ in quanto individuo di una più vasta società. Le narrazioni di Tondelli non sono quindi prive di una dimensione etica, ma i critici ne hanno più spesso parlato mettendone in luce quegli elementi che portano a descriverlo non solo come uno scrittore "generazionale", ma quasi "settoriale", nel senso che gli si attribuiscono luoghi, situazioni, modelli, e soprattutto un linguaggio tipico della sua generazione, se non addirittura di un determinato ambiente. Forse, questa interpretazione, benché limitativa, può essere accettata per una parte della sua produzione. Quando si prenda in considerazione Camere separate, quindi, il registro deve cambiare, in quanto questo romanzo di Tondelli non è semplicemente lo specchio letterario di una generazione "sballata". Ciononostante, a Camere separate sono state dedicate decine di recensioni, ma solo un numero ristretto di saggi critici. Fra questi ultimi, e poiché l'argomento di questo articolo è l'interazione fra viaggio e rinascita, non si può non tenere conto delle considerazioni a questo proposito fatte da Sonia Basili, la quale inizia a confrontarsi con almeno uno degli aspetti cognitivi del testo: "Camere separate è soprattutto la storia di una maturazione" (Basili, "Morte e rinascita," 20), e in modo pertinente sottolinea, fin dal titolo del suo articolo, la dinamica che lega il processo di morte e rinascita con quella del viaggio. Tuttavia, le conclusioni della Basili si indirizzano sostanzialmente all'importanza che il linguaggio assume in questa evoluzione, facendone in ultima analisi un calibro per la misura dell'aut-

${ }^{3} \grave{E}$ significativo, in questo senso, che quasi tutti i racconti di Altri libertini si concludano con partenze o ritorni. 
enticità delle emozioni narrate da Tondelli, istituendo così una dimensione autobiografica del romanzo. Pur partendo da presupposti simili, in questa sede considereremo il linguaggio come uno dei momenti costituenti della rinascita, e non come lo stadio ultimo di essa.

Proprio alcune riflessioni su una precisa scelta linguistica, forniscono lo spunto per guardare al testo un po' più da vicino. Viaggiare, spostarsi significa mettere un distacco fra il sé e ciò che quotidianamente lo circonda. Nel caso di Camere separate l'allontanamento è anche emotivo e si riflette in primo luogo in relazione al narrato. La distanza è quindi anche una cifra linguistica e stilistica; Leo, infatti, sceglie la terza persona per raccontarsi. Nonostante in tutto il romanzo il protagonista sia indicato con "lui", non c'è alcun dubbio, infatti, che il narratore implicito sia Leo stesso. La scelta grammaticale riflette la volontà del protagonista di prendere le distanze dalla vicenda, e gli permette contemporaneamente di parlare di una parte della propria identità in cui non si riconosce più. Il protagonista narra al lettore di un sé che è stato ma che ora non esiste più; da parte sua, il lettore non può che stare al gioco: "So di chi stai veramente parlando, ma se non vuoi dichiararti non importa, io ti ascolto comunque" divenendo così, fin dalle prime righe, complice di Leo. Questa complicità non può essere poi facilmente destituita, e il lettore é chiamato a partecipare della continua evoluzione del protagonista. L'esistenza stessa di questa complicità, non consente poi una lettura critica in chiave "generazionale," ma chiede piuttosto una risposta attenta alle problematiche più profonde che emergono dal testo e che sono relative, suggeriamo in questa sede, ad uno sviluppo della dimensione etica del romanzo. L'uso del pronome "lui", infine, sottolineando il bisogno di mettere una distanza, permette di porre l'accento su un altro aspetto del movimento, quello relativo alla fuga.

Prima di prendere in esame l'idea dell'evasione, in Camere separate, è però necessario fare delle distinzioni interne al romanzo stesso. Il rapporto viaggio-rinascita non si attua sempre attraverso una dinamica di causaeffetto, perché ad ogni viaggio non sempre consegue una rinascita. La narrazione infatti, che procede in modo assolutamente asistematico dal punto di vista cronologico, è caratterizzata dai continui spostamenti di Leo, sia in assenza che in presenza di Thomas. L'abolizione dello scandire tradizionale del tempo permette di enfatizzare due momenti in cui la fuga dalla propria quotidianità porta alla rigenerazione, e più precisamente: il viaggio mentale dovuto alla droga e quello fisico che egli intraprende in seguito alla morte di Thomas.

Durante la giovinezza, Leo, assumendo sostanze stupefacenti, aveva vissuto una specie di incubo ad occhi aperti in cui aveva capito di essere 
giunto al termine dell'adolescenza e di trovarsi alle soglie dell'età adulta. Un'esperienza molto simile viene rivissuta in seguito alla morte del compagno, non è un caso quindi che esse vengano accomunate alla fine del primo movimento:

Gli occhi che solo qualche ora fa hanno visto la morte già non guardano più nello stesso modo. Anni e anni prima, quando aveva poco più di vent'anni, forse era successo un avvenimento analogo. E lui si era accorto, nel crescere, che quel particolare fatto non era stato nient'altro che il superamento traumatico, violentissimo della barriera che lo teneva racchiuso nella sua adolescenza, nei suoi miti, nelle sue illusioni. Quasi improvvisamente si era reso conto di essere un uomo. Non era più il ragazzo non era più l'immortale. (Camere, 39)

Nel viaggio mentale causato dalla droga, Leo perde il dominio di se stesso, riesce a malapena a governare i propri atti e di certo non può controllare i propri pensieri e si trova a vivere un'esperienza allucinatoria ed extracorporale: "Improvvisamente si vide, anche se dall'esterno, anche se da molto distante" (Camere, 48). Le sue azioni, così come la sua mente, sono guidate da una forza che lui non riesce a controllare e, mentre il corpo sembra voler superare i limiti fisici del sensibile: "Non sentiva la fatica, non sentiva il battito del proprio cuore, né il respiro che gli gelava i denti. Non sentiva né il freddo né il caldo né il bene né il male. Era troppo abissalmente lontano." (Camere, 47), la mente vuole sfiorare l'assoluto, vuole pensare al di là delle epoche e dei luoghi, quasi annullarsi per poter finalmente comprendere l'universo di cui fa parte. Nel medesimo istante comincia a percepirsi come essere unico, ma multisfaccettato, ed intuisce il germinare di una nuova presenza interna alla sua vita:

Non si rendeva conto di quanto tempo fosse passato. Ma passato da quando? Quando era iniziata la sua storia? O quella dell'Altro? Ma chi era lui? Era profondamente se stesso, ma nello stesso tempo era nessuno. Nessuno. Senti di impazzire, una, dieci, cento milioni di volte. (Camere, 47)

II fatto di essere cosciente non gli è sufficiente a riprendere la padronanza mentale e fisica, la sua identità si annulla e contemporaneamente si moltiplica e lui non può fare altro che subire queste rapidissime evoluzioni: "Era un'individualità che soffriva nel divenire" (Camere, 49). Quella notte cominciata in un casolare e finita simbolicamente davanti al mare, perché appunto: "Tutto nasceva dal mare. E al mare lui era tornato" (Camere, 49), aveva rappresentato un punto di non ritorno, una morte e una rinascita. Il narratore è molto esplicito in questo senso: "Voleva morire e trovare la 
pace. Ma era già morto e sapeva che la pace non era nemmeno là." (Camere, 47), e ancora poco dopo: "Si vedeva come un feto abortito sballottato da un utero all'altro" (Camere 49).

Durante quel viaggio Leo aveva creduto di morire e per ritornare alla vita aveva dovuto cercare cose esterne al proprio corpo. Completamente sconvolto dalle allucinazioni aveva avuto bisogno del mondo esterno per riconfermare la propria esistenza, per questo motivo: "cercò di ancorarsi a quello che sentiva, che toccava con le sue mani che portava alla bocca e che aveva un sapore di acquitrino, di pantano di marcio ma che lui poteva ancora ricordare, situare in una visione "reale"" (Camere, 48). La necessità di toccare e sentire ciò che gli sta intorno, è determinata dal bisogno di definirsi come un corpo separato, staccato da ciò che lo circonda. Come nel caso di una nascita vera e propria, il corpo del feto deve staccarsi da quello della madre per poter esistere come singolo, così Leo avverte l'urgenza di toccare ciò che è al di fuori di lui per sentirsi soggetto distinto da ciò di cui fa parte. In altre parole, per poter rinascere Leo deve ridefinirsi come individualità fisica in primo luogo, questo gli permetterà in seguito di percepirsi come identità singola rispetto alla totalità.

La fisicità aveva giocato, in quel frangente un ruolo determinante, in quanto la ridefinizione della propria corporeità aveva riportato la mente ad una condizione normale. L'esperienza metacorporale era finita nel momento in cui il corpo aveva imposto i suoi limiti. Quello che gli fa riprendere coscienza è infatti uno dei sensi: "E fu un odore a riportarlo a casa" (Camere, 51), e più precisamente "era l'odore della sua terra" (Camere, 51). Nel momento stesso in cui riesce a distinguere l'odore della terra che gli è familiare, riconoscendo, perciò, e confermando così le proprie radici, Leo comincia a pensare al futuro: "Avrebbe dovuto ricostruirsi, giorno dopo giorno [...]. Era in un certo senso, una persona nuova; o forse era semplicemente morto un Leo e ne era nato uno diverso" (Camere, 51-52). Il protagonista capisce che tipo di uomo sarebbe diventato nel corso degli anni, e da quel momento comincia a costruirsi un po' alla volta:

Così giorno dopo giorno, anno dopo anno, il nuovo Leo era cresciuto e si era rafforzato. Il suo sguardo era profondamente cambiato. Era la stessa persona ma portava in sé la traccia sanguinante di un aborto, di un Leo che si era bruciato. Conservava nel profondo le cicatrici e le fratture dell'altro. (Camere, 52)

La narrazione è decisamente proiettata verso il futuro ma il Leo che era uscito da quell'esperienza era consapevole che la propria identità non era univoca e che gli sviluppi evolutivi si realizzavano attraverso nette rotture, 
fratture irrimediabili, aborti appunto. La cruda scelta semantica del narratore nel descrivere le sensazioni e le immagini allucinatorie del bad trip dà a tutto questo episodio un sapore di auto-procreazione, dagli echi quasi sacrali: "Stava incarnandosi di nuovo, in riva a quel mare, nella sua storia. Stava discendendo in sé [...] (Camere, 50). Egli arriva, pur senza esplicitarlo, a percepirsi come padre e figlio di se stesso, capacitandosi di questo rapporto genitoriale perchè per la prima volta guarda a sé stesso proiettandosi nel futuro e capisce " $[\ldots]$ che la sua prima giovinezza era finita con la consapevolezza dolorosa di essere uno dei miliardi di esseri in gioco" (Camere, 51). Leo comprende che vivere significa essere parte di un sistema, e non può più vivere come una monade esistente per sé ed in sé, egli: "[...] avrebbe imparato le regole" e ad esse si sarebbe adeguato consapevole, ora, che il suo agire deve essere responsabile in primo luogo nei confronti di sé stesso. Leo non si rinnega, ma cambia: "Conservava nel profondo le cicatrici e le fratture dell'altro (il corsivo è mio). E ogni tanto [...] le riapriva e le cambiava di luogo e solo in questo spostamento della frattura primaria e assoluta lui riuscì ad avere un po' di pace" (Camere, 52). Il suo nuovo sé mantiene le tracce dell'altro nella misura in cui queste gli consentono di avere pace, nella misura in cui cioè, non rappresentano un limite, o un pericolo per il nuovo Leo che sta nascendo. Il rapporto non abusivo nei confronti dell'Altro postulato da Lévinas si realizza qui nell'identità del protagonista, il quale riconosce nel proprio sviluppo un momento di cambiamento in cui vi è il germinare di un'identità in formazione, non ancora definibile, ma che sente come certamente diversa rispetto alla precedente.

Se la scioccante esperienza sulla spiaggia gli aveva insegnato che quella del cambiamento è una necessità, ciò nondimeno il dolore che comporta, e che Leo ora conosce profondamente, è tale da giustificare il desiderio di fuggire. Una parte dell'uomo che nasce in quella spiaggia, muore in una notte definita: "gravida del pensiero di Thomas che sta morendo"(Camere, 52). Si ritornerà fra breve sul valore di quel gravida, per ora soffermiamoci sul fatto che quella notte Leo riconosce di trovarsi in una situazione simile a quella di tanti anni prima. La morte del compagno diviene infatti il pretesto per lo spostamento fisico che caratterizzerà tutto il secondo movimento: in seguito ad essa Leo ha bisogno di allontanarsi da tutto ciò che lo circonda ogni giorno, la casa, la città, gli amici. Il viaggio diviene una fuga. Non è un caso che la morte di Thomas sia stata definita il "pretesto" per mettersi in viaggio, la ragione vera, infatti, non è tanto il bisogno di allontanarsi dalla morte di Thomas, quanto quello di distanziarsi dalla propria quotidianità, e di conseguenza da una parte della sua identità. La morte di Thomas mette termine ad una fase della vita del protagonista, muore per- 
ciò un po' anche Leo stesso; in particolare muore il Leo che aveva saputo trovare l'equilibrio statico ${ }^{4}$ delle "camere separate." Il viaggio allora non è solamente uno spostamento fisico, ma da intendersi in modo quasi metafisico, divenendo un'evasione dal sé. Ancora una volta è Emmanuel Lévinas a fornirci la chiave di lettura di questo particolare momento, descrivendo quale sia l'impulso che fa muovere Leo:

Lévasion au contraire met en question précisément cette prétendue paix avec soi, puisq'elle aspire a briser l'enchaînement du moi au soi. Dans l'évasion le moi se fuit non pas en tant qu'opposé a l'infini de ce qu'il n'est pas ou de ce qu'il ne deviendra pas, mais au fait meme qu'il est ou qu'il devient. (Lévinas, De l'évasion, 74)

Ciò che il desiderio di evadere mette in discussione è proprio quel legame interno al sé, che lo incatena ad una sola identità. L'equilibrio che questo desiderio mira a rompere, è quello raggiunto fra il me ed il sé, rappresentante quest'ultimo del fatto stesso di esistere come identità unica, che si sviluppa e diviene, ma resta fondamentalmente una. Il sé ha bisogno di sciogliersi da questo legame che risulta limitante e che di conseguenza provoca disagio, e l'unico modo per poterlo fare è seguire l'impulso di rompere l'equilibrio interno.

Allo stesso modo Leo, fugge non perché rifiuti di affrontare la morte di Thomas, ma in quanto questa lo costringe ad un'unica evoluzione o movimento possibile, e quindi ad una sola identità: quella di Leo senza Thomas. La morte, essendo un atto assoluto, non gli lascia alternative e l'unica cosa che Leo riesce a fare, perciò, è evadere da questa identità che oltretutto non ha scelto, ma che si trova costretto a subire. La perdita di punti di riferimento relativa alla nuova situazione mette Leo in una condizione di debolezza, ma che non gli è, a ben vedere, completamente nuova. Egli infatti sa come reagire:

Si sente prigioniero (il corsivo è mio) del buon senso dei suoi amici che mai si metterebbero in viaggio senza programmare né prenotare. [...] In realtà mente perché avverte tutta l'esilità delle sue motivazioni. Sa solamente che deve mettersi in viaggio. Non sa più cosa fare di se stesso. (Camere, 57)

Il protagonista prenderà coscienza solo in un secondo momento che questo suo bisogno di mettersi in viaggio è una vera e propria fuga: "Sta

${ }^{4}$ A questo proposito vedi anche quanto affermato da Enrico Minardi nel volume Pier Vittorio Tondelli, 52. 
scappando per tutta l'Europa dall'orrore della perdita di Thomas. [...] Sta scappando dalla morte" (Camere, 68). E' sintomatico che si parli di "perdita di Thomas," ma che sia invece molto più generalmente indicato che sta scappando dalla morte. Apparentemente non dovrebbe esserci bisogno di aggiungere "di Thomas," che suonerebbe pleonastico dato il contesto; in realtà è tanto più significativo proprio perché passato sotto silenzio. La ragione per cui il viaggio si definisce come momento metafisico sta proprio in questa omissione: il movimento trascende la realtà effettiva in quanto Leo, oltre che dalla perdita di Thomas, fugge dalla propria morte; non quella fisica, evidentemente, ma dalla fine di una parte della sua identità. Ancora una volta, come era già successo sulla spiaggia, Leo deve ridefinirsi come individuo: egli sa che una nuova fase della sua vita sta iniziando e che una nuova parte di sé si dovrà sviluppare. Si ripropone qui la dinamica di sviluppo in cui l'affermazione della nuova identità, del nuovo "altro" che sta nascendo, non può risolversi semplicemente con la soppressione del Leo che smette di essere la notte in cui muore Thomas.

Lo sconvolgimento provocato dalla scomparsa del compagno gli fa capire che la sua storia ha toccato nuovamente un punto di non ritorno, e così come era successo nella spiaggia molti anni prima, anche in questo momento Leo è costretto a proiettarsi verso il futuro. Quanto affermato in precedenza in relazione al significato della morte di Thomas, vissuta come evento che scaraventa Leo in un domani che non ha programmato, consente di affermare che quella notte è gravida perchè c'è un nuovo Leo che sta nascendo. Traspare anche in questo caso un'apertura verso il futuro, ma a differenza del bad trip sulla spiaggia, questa volta Leo è consapevole che un nuovo sé sta per emergere; quindi, se allora solo alla fine del viaggio aveva capito che tipo di uomo sarebbe divenuto, similmente ora ha bisogno di viaggiare, perché solo così saprà come sarà il nuovo Leo.

Il viaggio attraverso l'Europa che inizia dopo la morte di Thomas, e che apre il secondo movimento, non sarà che l'inizio del lungo percorso, fatto di tanti diversi spostamenti, che porteranno alla rigenerazione. Il viaggio comincia come "[...] la sua spedizione ai confini del corpo di Thomas" (Camere, 58), e parte d'autunno perché, scrive Tondelli in Un week-end postmoderno:

Solo ora rileggendo Roland Barthes alla voce 'fading' (svanimento) capisco perché mi è capitato di associare l'idea del viaggio autunnale all'idea di perdita di voce, quindi al silenzio. Il 'fading' dell'altro è racchiuso nella sua voce. La voce sostiene, rende leggibile, e per così dire, realizza l'evanescenza dell'essere amato, poiché è alla voce che tocca di morire. (Tondelli, Week-end postmoderno, 380) 
Che questo commento sia strettamente legato a Camere separate ̀̀ dimostrato dal fatto che le pagine che seguono, in Un week-end postmoderno, sembrano la prima stesura del romanzo. Quello in seguito alla scomparsa di Thomas non può che essere un viaggio autunnale perchè va 'verso il silenzio'. L'autore qui sembra voler fare un po' lo sgambetto: perché intitolare "Verso il silenzio" il primo movimento e non il secondo, quello in cui effettivamente Thomas 'fades'? La risposta è data dalla consapevolezza che nel secondo capitolo c'è già un altro movimento che sovrasta il fading, ovvero l'atto di scavare nella vita del protagonista, nel suo mondo appunto, da cui il titolo "Il mondo di Leo", per mettere in atto una specie di archeologia personale che lo porterà alla rinascita.

La scelta relativa ai titoli, conferma che in Camere separate, l'allontanamento, o fuga, non è da intendersi con valore esclusivamente negativo: lo slancio verso l'altrove provato dal soggetto non mira unicamente a soddisfare un bisogno di rottura intesa come annullamento rispetto a quello che precede, perché ad esso corrisponde anche una apertura verso il futuro. Questo significa che lo spostamento del sé verso ciò che ancora non conosce non è interpretabile limitatamente come cessazione del momento antecedente il movimento, in quanto la frattura è anche portatrice di uno sviluppo ulteriore, di una rinascita. Il trauma della morte di Thomas definisce il domani di Leo come irrimediabilmente diverso rispetto ad oggi o ieri, perciò egli non ha altra scelta e deve cominciare a ricostruire se stesso in funzione di questo stacco. E' stato precedentemente affermato che la parte di Leo che muore è quella che aveva saputo ideare l'equilibrio statico delle "camere separate". La relazione vissuta in questi termini, infatti, era immobile, non vi era una sostanziale differenza fra il passato ed il presente, perché i due protagonisti condividevano momenti di un rapporto che restava fondamentalmente immutato nelle sue modalità. Tanto è vero che anche l'inserimento di Susan, pur provocando inizialmente un forte turbamento in Leo, non cambia, di fatto, la relazione fra $\mathrm{i}$ due. Questa immobilità viene infranta inaspettatamente dalla morte del compagno che, come abbiamo visto, costringe Leo a pensare ad un solo futuro possibile, quello in cui Thomas è morto.

Lévinas opera una netta distinzione fra il momento della fuga e quello dello slancio "l'élan vital” (Lévinas, De l'évasion, 71). Nella fuga il soggetto obbedisce esclusivamente al bisogno di allontanarsi da ciò che lo circonda senza darsi un punto d'arrivo o una meta; nello slancio vitale, invece, il soggetto va verso qualche cosa, che può essere indefinito o addirittura sconosciuto, ma è sempre un obiettivo finale. Quando parte, Leo non sa ancora in quale paese o città si stia dirigendo, perché lo scopo del viaggio 
non è in nessun modo correlato con il mondo esterno: "Non ha fissato una meta precisa. Ha intenzione di fare un viaggio lento, in treno, attraverso l'Europa" (Camere, 58). Egli non è un normale turista che si reca a visitare un paese straniero, perché il suo è un viaggio dentro il proprio dolore e, insieme, un viaggio di ritorno alle proprie radici. Fra le tappe di questo lungo spostamento, che si sviluppa fra Europa e Nord America, c'è infatti anche il ritorno al paese d'origine e alla casa materna. Durante la permanenza al paese natio, attraverso un indiscriminato alternarsi di ricordi vicini e lontani, che vanno dalla sua infanzia ai più recenti viaggi con Thomas, Leo, sembra girare con meticolosa pazienza, il coltello nella piaga. Questo atto gli è necessario perchè le cicatrici, come si è già visto al termine del viaggio adolescenziale, gli servono a ricordare ciò che è stato, a trovare pace, e soprattutto a non rinnegarsi.

Quella alla casa dei genitori, però, è solo una tappa della sua fuga che finisce, in modo simbolicamente violento sul piano fisico, in una serata al Blue Boy, un bar di Washington dal nome più che appropriato. Leo, viene trascinato, quasi suo malgrado, in un'esperienza sadomasochista con uno sconosciuto, il boy, durante la quale contro la sua volontà, gli viene somministrata della droga. Fin da subito le immagini del rapporto sessuale si intervallano con quelle allucinatorie di un ospedale in cui Leo, prima vecchio e poi bambino, aspetta di essere operato. In una condizione di semiincoscienza Leo dà un correlativo mentale alle sensazioni che il suo corpo sta provando, ed il filo conduttore fra i due momenti è costituito dal dolore:

Gli serra la gola con un collare. Leo ha paura di soffocare. Sente improvvisamente la testa scoppiare, ingigantirsi di sangue bollente, suda, la vista gli si affievolisce e il respiro si fa un rantolo sordo.

Quando era venuto il momento di intubarlo, nel gelo della sala operatoria, lui era ancora cosciente. Era vivo in un corpo morto. Imprigionato. (Camere, 159)

I due livelli di percezione, quello mentale e quello fisico diventano da questo punto della narrazione in poi, molto intricati, rendendo difficilmente distinguibile cosa sia frutto di un'allucinazione, e cosa invece sia dovuto alle: "pratiche di un onesto torturatore" (Camere, 160). La fusione fra vissuto e immaginato segna tutto il racconto dell'episodio, fino a che, quando il dolore sembra raggiungere un livello insopportabile, Leo percepisce con netta chiarezza di essere, ancora una volta, arrivato ad una frattura. In quel momento la definizione fra fisico e mentale diviene estremamente precisa perché il protagonista è cosciente del processo in atto, è consapevole che quello è: "il dolore di un corpo che ancora non conosce, come 
invece il suo cervello, il proprio punto di rottura" (Camere, 160). Leo è in grado di riconoscere il punto di rottura del suo cervello, perché era stato raggiunto durante il bad trip giovanile. Anche in questo caso l'evoluzione della propria identità viene percepita ed identificata attraverso l'ormai nota dinamica di morte e rigenerazione. A morire, qui, è il Leo nato nella notte gravida della morte di Thomas:

Il disagio [...] è qualcosa che lui non vuole abbandonare come se finalmente tutto il dolore che da anni sta provando, da quando Thomas è morto, da quando lui, Leo, è nato, si concretasse in quel lettino, in quel corpo serrato, ai testicoli e alla gola da un laccio di cuoio (Camere, 160).

Da quel dolore Leo rinasce, l'episodio si conclude, infatti, con il protagonista che rassicura il boy: "[...] con una voce che non avrebbe mai pensato di avere: quella del bambino-Leo. Una voce stridula, acuta, femminile, un vagito sepolto nel profondo del suo dolore e che il dolore ha messo di nuovo al mondo" (Camere, 161). Ancora una volta la fisicità ha un ruolo determinante, anche se con modalità leggermente diverse. Durante il viaggio sulla spiaggia, il protagonista aveva vissuto un'esperienza eminentemente mentale, ed il corpo aveva avuto una funzione normalizzante; nel caso della serata a Washington, trattandosi di un rapporto sessuale, l'equilibrio corpo-mente è molto più fragile, ma sarà ancora una volta la corporeità a ricostituire la normalità. Leo resterà negli Stati Uniti ancora per poche setrimane, ma simbolicamente il viaggio finisce quella sera a Washington perché lì termina il percorso che Leo intraprende all'inizio del secondo movimento, quello che va "oltre i confini del corpo di Thomas" (Camere, 57), ma che, in modo simile a quanto era avvenuto durante il bad trip, termina laddove Leo raggiunge il suo limite fisico.

A Washington termina anche la fuga di Leo, e pian piano, inconsapevolmente, comincia lo "slancio vitale" verso la nuova fase della sua vita. Le conseguenze della serata al Blue Boy non sono immediate. Il narratoreLeo accusa il colpo, ma ha bisogno di tempo per assimilarne i risvolti, e la narrazione si interrompe bruscamente. Il bisogno di procrastinare qualsiasi riflessione in merito al rapporto sadomasochista si risolve quindi, in una negazione temporanea di commentare o soffermarsi sull'accaduto. Dovrà passare del tempo, prima che Leo possa pensare all'episodio di Washington e al suo significato. È una presa di coscienza che avanza lentamente, per piccole fasi, assumendo in un primo momento i caratteri della colpa:

E allora pensò che anche lui aveva sepolto, in un certo senso, Thomas. E che, sia lui sia il vecchio, erano degli assassini che in un modo o in un 
altro avevano controllato fino alla fine la vita della persona che più amavano. Fino a deporre nella fossa il corpo che avevano creato. Allora Leo sente che questa necessità di sadomasochismo non è un impulso estraneo, ma forse la perversione più pura che abbia mai provato, quella più sincera. Perchè lui è un torturatore ed è la vittima designata di quell'aguzzino che porta il suo stesso nome. (Camere, 164)

Identificandosi contemporaneamente come vittima e carnefice egli mette in discussione il Leo nato da quell'episodio. È uno sviluppo che inizia perciò con un segno negativo, ovvero con la rassegnazione all'autodistruzione. Ma questa non è che la prima fase.

Il terzo movimento, quello che dà anche il titolo al libro, è quello in cui il processo di rinascita si completa. Al sentimento autodistruttivo subentra, dopo il rientro in Italia, l'accettazione di quella parte della propria identità che non voleva riconoscere: "Si rende conto che dovrà accettarlo come una parte di sé, qualcosa [...] che ha fatto affiorare alla coscienza ciò che lui si è sempre negato: cioè che è impastato non solo di bene, come sosteneva Thomas, ma anche di colpa" (Camere, 190). Il comune buon senso vuole che il modo migliore per superare un problema sia ammettere di averne uno. Quali conseguenze ha, allora, per il protagonista l'ammissione della colpa, la consapevolezza di essere fatto di 'male' oltre che di 'bene'? Il problema da risolvere, nel caso di Leo, è la ridefinizione della propria identità, non solo in seguito alla morte di Thomas, ma di ciò che in conseguenza di essa egli scopre di se stesso. Dopo Washington ha bisogno di "digerire l'impatto che quell'atto sta avendo sulla sua solitudine" (Camere, 190), e la solitudine è proprio il nodo da districare.

La fuga gli aveva consentito di rompere il legame interno fra il me ed il sè, evitando una coatta evoluzione in un'unica possibile identità. Essa è stata definita come momento positivo, in quanto non è solo rottura, ma apertura a ciò che segue. Quella di Leo era stata una fuga solitaria in cui luoghi e persone avevano giocato un ruolo poco rilevante. La scelta di non voler più amare aveva comportato anche un inaridimento degli altri rapporti sociali: "Allora capisce che la rinuncia all'amore, che in un qualche modo si è imposto, lo sta uccidendo, lo sta separando sempre di più dagli altri confinandolo in una zona incattivita e sterile dalla quale è sempre più difficile uscire" (Camere, 191). Perpetrare la sua solitudine, più che al superamento del lutto, lo aveva portato a cercare un'autodefinizione mediante l'annullamento delle relazioni con altre persone. Evitare gli altri diviene, in ultima analisi, un modo per evitare una dinamica di auto-riconoscimento, eludendo l'individuazione di sé rispetto alla totalità. La ricerca di solitudine quindi non va interpretata come una fuga, ma al contrario come un momento 
di stasi rispetto allo slancio vitale. La scelta di isolarsi, in questo senso, può essere concepita anche come una forma di passività, in quanto Leo non prende iniziative, e lascia che gli eventi prendano il loro corso senza cercare di intervenire attivamente: "[...] anche perché a Leo è sempre risultato più facile caratterialmente, rinunciare piuttosto che insistere, sparire invece di continuare" (Camere, 192). Nonostante la confessata mancanza di capaci-tà reattiva, Leo sa che il confronto con il mondo esterno è quello che gli è necessario per rinascere. Sulla spiaggia aveva avuto bisogno di toccare il fango e sentire l'odore della sua terra per confermare la propria esistenza, ovvero la propria individualità in quanto essere separato, in questo caso ha bisogno di un riscontro sociale che gli permetta di darsi una nuova collocazione all'interno di quel mondo di cui poco per volta sta tornando a fare parte. La ricostruzione continua: "[...] perché quello che ora cerca non è un compagno, ma un suo nuovo posto nel mondo" (Camere, 202). La propria ricollocazione "nel mondo" prelude alla rinascita definitiva della parte di Leo che era morto con Thomas, che avviene, e c'era da aspettarselo, durante un viaggio. Mentre si trova a Quebec per un convegno internazionale su Jack Kerouac, Leo sente rinascere il desiderio: "Poi improvvisamente qualcosa di terribilmente bello nasce anche per lui, con la meraviglia di un seme che fiorisce dopo quattro lunghi anni di cure continue, di aridità, di siccità, di diluvi" (Camere, 215).

I momenti del romanzo analizzati nello specifico, il bad trip giovanile ed il viaggio in seguito alla morte di Thomas, sono accomunati, inoltre, da un altro importante elemento: la passività. Anziché portare a conclusione la lettura fin qui proposta di Camere separate, il concetto di passività come momento cognitivo, apre nuove possibilità critiche. Non potendo in questa sede affrontare compiutamente questo argomento, ci si limiterà ad un breve accenno non esaustivo ma utile ad individuare un complementare sviluppo etico di questo romanzo. Nella sua premessa a Dall'esistenza all'esistente, Pier Aldo Rovatti, uno dei più importanti commentatori di Lévinas, dà una stimolante interpretazione di questa categoria: "È certamente una nozione di positività, la passività in Lévinas, dal momento che è la chiave di accesso al rapporto etico", e più oltre: "Lévinas tiene fermo al soggetto: esso si denuclea, si scava in se stesso, diviene la passività più passiva, ma si mantiene" (Lévinas, Dall'esistenza all'esistente, 68). Pur non volendosi soffermare troppo sulla comunque sorprendente vicinanza terminologica rispetto alla prima citazione di Tondelli, interessa qui la concezione di una passività intesa come dinamica positiva. Nei due episodi più forti del romanzo, Leo non ha il controllo della situazione, da giovane a causa degli effetti della droga, a Washington perchè legato e quindi semi-immobilizza- 
to. In entrambi i casi egli è costretto a subire passivamente gli eventi. Questa passività è totale, tanto da fargli credere di soccombere, cioè di arrivare all'inattività per eccellenza; ma in entrambi i casi, il momento di passività ultima non precede il vuoto, la morte, bensì l'inizio della ricostruzione. La passività, anche se costretta, perchè è in primo luogo dovuta ad una costrizione fisica, è quella che consente a Leo di scavare all'interno di quell'identità che si sta evolvendo, di modificarsi pur mantenendosi come essere unico, dandogli così la possibilità di ridefinirsi come individualità. Il risvolto etico, inoltre, si articola non semplicemente nella diversa consapevolezza di sé da parte del protagonista, ma nelle conseguenze pratiche che derivano da quel momento di "passività più passiva", in particolare nel suo modo di affrontare il proprio domani. All'iniziale rifiuto di Leo di sentirsi partecipe di un'umanità da cui preferisce isolarsi, seguirà invece la riscoperta della possibilità di rapporti umani più intensi, ma soprattutto sentirà di nuovo risorgere la disponibilità ad amare, come avviene qualche tempo dopo in un bar di Quebec. Il romanzo, però, si conclude con una nota agrodolce: la consapevolezza che la sua vita sta per finire, è immediatamente preceduta $\mathrm{dal}$ risorgere della disponibilità sentimentale: "E contento perchè ha sentito rinascere la disponibilità" (Camere, 216).

In conclusione si è esaminato come nel romanzo di Tondelli la dinamica morte-rinascita, filtrata attraverso la tematica del viaggio, sia mentale che fisico, metta in atto un meccanismo che va al di là dello spostamento in quanto tale, diventando piuttosto un percorso auto-rigenerativo. I tre capitoli-movimenti descrivono un percorso cognitivo personale, in cui il protagonista, ripercorrendo le tappẹ fondamentali della sua vita, deve ridefinire se stesso. Leo si percepisce infatti come soggetto in fieri in cui distingue la netta separazione fra il sé che era e quello che sta diventando. I risvolti di questo percorso personale sono di carattere etico in quanto, come proposto da Lévinas, Leo non può semplicisticamente rinnegare l'uno o l'altro, ma deve mettere in atto una mediazione che gli permetta di accettare il nuovo sé che sta nascendo, nella consapevolezza di essere responsabile nei suoi confronti.

Egli individua gli episodi che hanno rappresentato un vero e proprio punto di non ritorno: il bad trip sulla spiaggia e la morte di Thomas. Durante il viaggio mentale causato dalla droga, Leo ha creduto di morire e, in parte, è realmente morto, perché in quel momento ha sentito che la sua adolescenza finiva ed incominciava l'età adulta. Il ricordo di quella traumatica esperienza riemerge, non a caso, la notte in cui Thomas muore, ovvero quando il protagonista si trova nuovamente a dover affrontare la fine di una fase della sua vita. Leo, a quel punto, sa che la sola soluzione 
possibile per lui è mettersi in viaggio. Ritornando alla casa dei genitori, ma soprattutto viaggiando fra Europa e Nord America, egli vuole fuggire dall'univoca realtà in cui la morte di Thomas lo proietta. Allontanandosi e mettendosi in una condizione di straniero in una realtà semisconosciuta, egli prende le misure del nuovo sé, ed infine rinasce. L'esperienza al Blue Boy lo metterà in una situazione al limite delle sue possibilità psico-fisiche, come era accaduto nella spiaggia tanti anni prima. La fuga che era iniziata con la morte di Thomas termina in quel momento, e impercettibilmente, con un movimento molto lento, ricomincia anche la rinascita, che si completerà durante un viaggio a Quebec durante il quale sentirà rinascere l'interesse sentimentale.

Tondelli ha affermato che un'importante livello di lettura in Camere separate è quello del 'futuro anteriore' indicando con quest'ultimo lo svolgimento ulteriore, rispetto al narrato, di cui ogni movimento sembra farsi portatore non concludendosi in ciò che viene detto ma creando una specie di ponte verso il futuro e verso le differenti possibilità che questo porta con sé. Il futuro 'anteriore' è anche il tempo delle ultime pagine, il cui svolgimento ulteriore è quello che porta alla conclusione della vita stessa, "Allora saprà, con una determinazione anche commossa e disperata, che non c'è più niente da fare." (Camere, 216).

\section{McGill University}

\section{Opere Citate}

Basili, Sonia. "Morte e rinascita nelle esperienze erratiche di Pier Vittorio

Tondelli." La giovane narrativa italiana 8 (1992) : 19-40.

Lévinas, Emmanuel. Totalité et infini. Essai sur l'extériorité. Paris: Brodard et Taupin, 1971.

Lévinas, Emmanuel. De l'évasion. Montpellier: Fata morgana, 1982.

Minardi, Enrico. Pier Vittorio Tondelli. Fiesole: Cadmo, 2003.

Panzeri, Fulvio. Picone, Generoso. Pier Vittorio Tondelli. Il mestiere di scrittore. Roma: Napoli: Edizioni Theoria, 1997.

Rovatti, P. Aldo. "Premessa" a Emmanuel Lévinas, Dall'esistenza all'esistente. Genova: Marietti, 1986.

Tondelli, Pier Vittorio. Camere separate. Milano: Bompiani, 2001.

Tondelli, Pier Vittorio. Rimini. Milano: Bompiani, 1985.

Tondelli, Pier Vittorio. Un week-end postmoderno. Milano: Bompiani, 1993. 